C. Dharuman* and E. Thandapani

\title{
Oscillation of Solutions of Nonlinear Difference Equation With a Super-linear Neutral Term
}

https://doi.org/10.1515/msds-2018-0004

Received May 2, 2017; accepted April 3, 2018

Abstract: This paper deals with the oscillation of solutions of certain class of neutral difference equation

$$
\Delta\left(a_{n} \Delta\left(x_{n}+p_{n} x_{n-k}^{\alpha}\right)\right)+q_{n} x_{n+1-l}^{\beta}=0,
$$

where $\alpha$ and $\beta$ are ratio of odd positive integers. New sufficient conditions are obtained for the oscillation of studied equation and examples illustrating the main results are provided.

Keywords: Oscillation, difference equation, nonlinear, super-linear neutral term

MSC: 39A11

\section{Introduction}

In this paper, we are concerned with the nonlinear neutral difference equation of the form

$$
\Delta\left(a_{n} \Delta\left(x_{n}+p_{n} x_{n-k}^{\alpha}\right)\right)+q_{n} x_{n+1-l}^{\beta}=0, n \geq n_{0},
$$

where $n_{0} \in \mathbb{N}=\{0,1,2, \ldots\}$, subject to the following conditions:

$\left(C_{1}\right)\left\{a_{n}\right\}$ is a positive real sequence for all $n \geq n_{0}$;

$\left(C_{2}\right)\left\{p_{n}\right\}$ and $\left\{q_{n}\right\}$ are nonnegative real sequences for all $n \geq n_{0}$;

$\left(C_{3}\right) 0 \leq p_{n}<1$ for all $n \geq n_{0}$;

$\left(C_{4}\right) k$ is a positive integer, and $l$ is a nonnegative integer;

$\left(C_{5}\right) \alpha \geq 1$, and $\beta$ are ratio of odd positive integers.

Let $\theta=\max \{k, l\}$. By a solution of equation (1.1), we mean a real sequence $\left\{x_{n}\right\}$ defined for all $n \geq n_{0}-\theta$, and satisfies equation (1.1) for all $n \geq n_{0}$. A nontrivial solution of equation (1.1) is said to be oscillatory if the terms of the sequence are neither eventually positive nor eventually negative, and nonoscillatory otherwise.

In recent years there is a great interest in studying the oscillatory and asymptotic behavior of solutions of various classes of difference equations, see, for example [1-4, 6-10] and the references cited therein. In particular in [7], the authors considered the equation of the form (1.1) and obtained criteria for the oscillation of equation (1.1) under the conditions either

$$
\sum_{n=n_{0}}^{\infty} \frac{1}{a_{n}}=\infty
$$

${ }^{\star}$ Corresponding Author: C. Dharuman: Department of Mathematics, SRM University, Ramapuram Campus, Chennai - 600 089, India, E-mail: cdharuman55@gmail.com

E. Thandapani: Ramanujan Institute for Advanced Study in Mathematics, University of Madras, Chennai - 600 005, India, E-mail: ethandapani@yahoo.co.in 
or

$$
\sum_{n=n_{0}}^{\infty} \frac{1}{a_{n}}<\infty
$$

for the case $0<\alpha \leq 1$. In [9], the authors investigated the oscillatory behavior of equation (1.1) under the assumption (1.2), and $\alpha>1$. In order to solve the problem completely we examine the other case (1.3), which appears to be more difficult than the former. To accomplish this is the main purpose of the present paper. Thus the results obtained in this paper are new, and complement to that of in $[3,6-10]$.

\section{Oscillation Results}

In this section, we obtain sufficient conditions for the oscillation of all solutions of equation (1.1) under the assumption (1.3). Note that from the hypotheses, it is enough to state and prove the results for the case $\left\{x_{n}\right\}$ is eventually positive since the proof for the opposite case is similar.

In the following, for convenience we denote

$$
\begin{aligned}
z_{n} & =x_{n}+p_{n} x_{n-k}^{\alpha}, \\
A_{n} & =\sum_{s=n}^{\infty} \frac{1}{a_{s}}, \text { and } R_{n}=\sum_{s=N}^{n-1} \frac{1}{a_{s}}
\end{aligned}
$$

where $n \geq N \geq n_{0}$.

Lemma 2.1. Let $\left\{x_{n}\right\}$ be an eventually positive solution of equation (1.1). Then the corresponding function $\left\{z_{n}\right\}$ satisfies one of the following two cases for all sufficiently large $n$ :

1. $z_{n}>0, \Delta z_{n}>0, \Delta\left(a_{n} \Delta z_{n}\right) \leq 0$;

2. $z_{n}>0, \Delta z_{n}<0, \Delta\left(a_{n} \Delta z_{n}\right) \leq 0$.

Proof. The proof is similar to that of Lemma 2.1 of [7], and hence the details are omitted.

Lemma 2.2. Let $\left\{x_{n}\right\}$ be an eventually positive solution of equation (1.1) and the corresponding function $\left\{z_{n}\right\}$ satisfies Case(1) of Lemma 2.1. Then there exists an integer $N\left(N \geq n_{0}\right)$ such that for every constant $M>0$

$$
x_{n} \geq\left(1-M^{\alpha-1} p_{n} R_{n-k}^{\alpha-1}\right) z_{n}
$$

for all $n \geq N$.

Proof. From Case (1) of Lemma 2.1, we have $a_{n} \Delta z_{n}$ is decreasing and

$$
z_{n}=z_{N}+\sum_{s=N}^{n-1} \frac{a_{s} \Delta z_{s}}{a_{s}} \geq R_{n} a_{n} \Delta z_{n},
$$

which implies

$$
\Delta\left(\frac{z_{n}}{R_{n}}\right) \leq 0 \text { for all } n \geq N .
$$

On the other hand, from the definition $z_{n}$, we have $z_{n} \geq x_{n}$, and

$$
\begin{aligned}
x_{n} & \geq z_{n}-p_{n} z_{n-k}^{\alpha} \geq\left(1-p_{n} z_{n-k}^{\alpha-1}\right) z_{n} \\
& \geq\left(1-M^{\alpha-1} p_{n} R_{n-k}^{\alpha-1}\right) z_{n}
\end{aligned}
$$

where we have used $\frac{z_{n}}{R_{n}}$ is decreasing and $\frac{z_{n}}{R_{n}} \leq M$ for $M>0$. This completes the proof. 
Lemma 2.3. Let $\left\{x_{n}\right\}$ be an eventually positive solution of equation (1.1) and the corresponding function $\left\{z_{n}\right\}$ satisfies Case(2) of Lemma 2.1. Then there exists an integer $N\left(N \geq n_{0}\right)$ such that for every constant $K>0$

$$
x_{n} \geq\left(1-\frac{K^{\alpha-1} p_{n} A_{n-k}^{\alpha}}{A_{n}^{\alpha}}\right) z_{n}
$$

for all $n \geq N$.

Proof. Since $a_{n} \Delta z_{n}$ is decreasing, we have

$$
\Delta z_{s} \leq \frac{a_{n} \Delta z_{n}}{a_{s}} \text { for } s \geq n .
$$

Summing the last inequality, we obtain

$$
0 \leq z_{n}+A_{n} a_{n} \Delta z_{n}, n \geq N
$$

which implies

$$
\Delta\left(\frac{z_{n}}{A_{n}}\right) \geq 0, n \geq N .
$$

On the other hand, from the definition $z_{n}$, we have $z_{n} \geq x_{n}$, and

$$
x_{n} \geq z_{n}-p_{n} \frac{z_{n-k}^{\alpha}}{A_{n-k}^{\alpha}} A_{n-k}^{\alpha} \geq z_{n}-p_{n} \frac{z_{n}^{\alpha}}{A_{n}^{\alpha}} A_{n-k}^{\alpha} \geq\left(1-p_{n} \frac{K^{\alpha-1}}{A_{n}^{\alpha}} A_{n-k}^{\alpha}\right) z_{n}
$$

where we have used (2.6) and $z_{n} \leq K$ for all $n \geq N$. This completes the proof

Theorem 2.4. Let $\beta>1$ and condition (1.3) be hold. If there exists a positive, nondecreasing real sequence $\left\{\rho_{n}\right\}$ such that

$$
\lim _{n \rightarrow \infty} \sup \sum_{s=N}^{n-1}\left[\rho_{s} q_{s}\left(1-M^{\alpha-1} p_{s+1-l} R_{s+1-k-l}\right)^{\beta}-\frac{a_{s-l}\left(\Delta \rho_{s}\right)^{2}}{4 \beta K_{1}^{\alpha-1} \rho_{s}}\right]=\infty,
$$

and

$$
\lim _{n \rightarrow \infty} \sup \sum_{s=N}^{n-1}\left[A_{s+1}^{\beta} q_{s}\left(1-\frac{K^{\alpha-1} p_{s+1-l} A_{s+1-k-l}^{\alpha}}{A_{s+1-l}^{\alpha}}\right)^{\beta}-\frac{\beta A_{s}^{\beta-1}}{4 M_{1}^{\beta-1} a_{s} A_{s+1}^{\beta}}\right]=\infty
$$

hold for all constants $M>0, M_{1}>0, K>0$ and $K_{1}>0$, then every solution of equation (1.1) is oscillatory.

Proof. Assume the contrary that equation (1.1) has an eventually positive solution $\left\{x_{n}\right\}$, that is, there is an integer $n_{1} \geq n_{0}$ such that $x_{n}>0, x_{n-k}>0$, and $x_{n-l}>0$ for all $n \geq n_{1}$. From the definition of $z_{n}$, we have $z_{n}>0$ for all $n \geq N \geq n_{1}$, where $N$ is chosen so that two cases of Lemma 2.1 hold for all $n \geq N$. We shall prove that in each case we are led to a contradiction.

Case(1). From equation (1.1) and (2.1), we have

$$
\Delta\left(a_{n} \Delta z_{n}\right)+q_{n}\left(1-M^{\alpha-1} p_{n+1-l} R_{n+1-l-k}^{\alpha-1}\right)^{\beta} z_{n+1-l}^{\beta} \leq 0, \quad n \geq N .
$$

Define

$$
w_{n}=\rho_{n} \frac{a_{n} \Delta z_{n}}{z_{n-l}^{\beta}}, n \geq N
$$

Then $w_{n}>0$ for $n \geq N$, and

$$
\begin{aligned}
\Delta w_{n} & =\rho_{n} \frac{\Delta\left(a_{n} \Delta z_{n}\right)}{z_{n+1-l}^{\beta}}+\Delta \rho_{n} \frac{a_{n+1} \Delta z_{n+1}}{z_{n+1-l}^{\beta}}-\rho_{n} \frac{a_{n} \Delta z_{n}}{z_{n+1-l}^{\beta} z_{n-l}^{\beta}} \Delta z_{n-l}^{\beta} \\
& \leq-\rho_{n} q_{n}\left(1-M^{\alpha-1} p_{n+1-l} R_{n+1-l-k}^{\alpha-1}\right)^{\beta}+\frac{\Delta \rho_{n}}{\rho_{n+1}} w_{n+1}-\frac{\rho_{n}}{\rho_{n+1}} w_{n+1} \frac{\Delta z_{n-l}^{\beta}}{z_{n-l}^{\beta}} .
\end{aligned}
$$


By Mean value theorem, we have

$$
\Delta z_{n-l}^{\beta} \geq \beta z_{n-l}^{\beta-1} \Delta z_{n-l},
$$

and using this in (2.10) we obtain

$$
\Delta w_{n} \leq-\rho_{n} q_{n}\left(1-M^{\alpha-1} p_{n+1-l} R_{n+1-l-k}^{\alpha-1}\right)^{\beta}+\frac{\Delta \rho_{n}}{\rho_{n+1}} w_{n+1}-\beta \frac{\rho_{n}}{\rho_{n+1}^{2}} \frac{w_{n+1}^{2}}{a_{n-l}} K_{1}^{\alpha-1}
$$

where we have used $a_{n} \Delta z_{n} \leq a_{n-l} \Delta z_{n-l}$ and $z_{n} \geq K_{1}>0$ for all $n \geq N$. Now, using the method of completing the square in (2.11), we obtain

$$
\Delta w_{n} \leq-\rho_{n} q_{n}\left(1-M^{\alpha-1} p_{n+1-l} R_{n+1-l-k}^{\alpha-1}\right)^{\beta}+\frac{a_{n-l}\left(\Delta \rho_{n}\right)^{2}}{4 \beta K_{1}^{\alpha-1} \rho_{n}}, n \geq N .
$$

Summing the last inequality from $N$ to $n$, one obtains

$$
\sum_{s=N}^{n}\left[\rho_{s} q_{s}\left(1-M^{\alpha-1} p_{s+1-l} R_{s+1-l-k}^{\alpha-1}\right)^{\beta}-\frac{a_{s-l}\left(\Delta \rho_{s}\right)^{2}}{4 \beta K_{1}^{\alpha-1} \rho_{s}}\right] \leq w_{N}
$$

which contradicts (2.7) as $n \rightarrow \infty$.

Case(2). From equation (1.1) and (2.4), we have

$$
\Delta\left(a_{n} \Delta z_{n}\right)+q_{n}\left(1-\frac{K^{\alpha-1} p_{n+1-l} A_{n+1-l-k}^{\alpha}}{A_{n+1-l}^{\alpha}}\right)^{\beta} z_{n+1}^{\beta} \leq 0, n \geq N
$$

where we have used $z_{n}$ is decreasing. Define

$$
u_{n}=\frac{a_{n} \Delta z_{n}}{z_{n}}, n \geq N .
$$

Then $u_{n} \leq 0$ for $n \geq N$. From (2.5) we have

$$
\frac{a_{n} \Delta z_{n}}{z_{n}} A_{n} \geq-1, n \geq N .
$$

Thus

$$
\frac{-a_{n} \Delta z_{n}\left(-a_{n} \Delta z_{n}\right)^{\beta-1} A_{n}^{\beta}}{z_{n}^{\beta}} \leq 1
$$

for $n \geq N$. So, by $\left(-a_{n} \Delta z_{n}\right)>0$ and (2.13), we have

$$
-\frac{1}{L^{\beta-1}} \leq u_{n} A_{n}^{\beta} \leq 0
$$

where $L=-a_{N} \Delta z_{N}$. From (2.13), we obtain

$$
\Delta u_{n}=\frac{\Delta\left(a_{n} \Delta z_{n}\right)}{z_{n+1}^{\beta}}-\frac{a_{n} \Delta z_{n}}{z_{n}^{\beta} z_{n+1}^{\beta}} \Delta z_{n}^{\beta}, n \geq N .
$$

By Mean value theorem, we have

$$
\Delta z_{n}^{\beta} \leq \beta z_{n+1}^{\beta-1} \Delta z_{n},
$$

and using this and (2.12) in (2.16) we obtain

$$
\Delta u_{n} \leq-q_{n}\left(1-\frac{K^{\alpha-1} p_{n+1-l} A_{n+1-l-k}^{\alpha}}{A_{n+1-l}^{\alpha}}\right)^{\beta}-\beta \frac{u_{n}^{2}}{a_{n}} z_{n}^{\beta-1}, n \geq N .
$$

Since $\frac{z_{n}}{A_{n}}$ is increasing there is a constant $M_{1}>0$ such that $\frac{z_{n}}{A_{n}} \geq M_{1}$ for $n \geq N$, and hence (2.17) yields

$$
\Delta u_{n} \leq-q_{n}\left(1-\frac{K^{\alpha-1} p_{n+1-l} A_{n+1-l-k}^{\alpha}}{A_{n+1-l}^{\alpha}}\right)^{\beta}-\beta \frac{M_{1}^{\beta-1} A_{n}^{\beta-1}}{a_{n}} u_{n}^{2}, n \geq N .
$$


Multiplying (2.18) by $A_{n+1}^{\beta}$ and then summing the resulting inequality from $N$ to $n-1$, one obtains

$$
\begin{aligned}
\sum_{s=N}^{n-1} A_{s+1}^{\beta} \Delta u_{s} & +\sum_{s=N}^{n-1} A_{s+1}^{\beta} q_{s}\left(1-\frac{K^{\alpha-1} p_{s+1-l} A_{s+1-l-k}^{\alpha}}{A_{s+1-l}^{\alpha}}\right)^{\beta} \\
& +\sum_{s=N}^{n-1} \frac{\beta M_{1}^{\beta-1} A_{s+1}^{\beta} A_{s}^{\beta-1}}{a_{s}} u_{s}^{2} \leq 0
\end{aligned}
$$

or

$$
\begin{aligned}
A_{n}^{\beta} u_{n}-A_{N}^{\beta} u_{N} & +\sum_{s=N}^{n-1} \frac{\beta A_{s}^{\beta-1}}{a_{s}} u_{s}+\sum_{s=N}^{n-1} \frac{\beta M_{1}^{\beta-1} A_{s+1}^{\beta} A_{s}^{\beta-1}}{a_{s}} u_{s}^{2} \\
& +\sum_{s=N}^{n-1} A_{s+1}^{\beta} q_{s}\left(1-\frac{K^{\alpha-1} p_{s+1-l} A_{s+1-l-k}^{\alpha}}{A_{s+1-l}^{\alpha}}\right)^{\beta} \leq 0
\end{aligned}
$$

which yields

$$
\sum_{s=N}^{n-1}\left[A_{s+1}^{\beta} q_{s}\left(1-\frac{K^{\alpha-1} p_{s+1-l} A_{s+1-k-l}^{\alpha}}{A_{s+1-l}^{\alpha}}\right)^{\beta}-\frac{\beta A_{s}^{\beta-1}}{4 M_{1}^{\beta-1} a_{s} A_{s+1}^{\beta}}\right] \leq \frac{1}{L^{\beta-1}}+A_{N}^{\beta} u_{N}
$$

when using (2.15). This contradicts (2.8) as $n \rightarrow \infty$, and the proof is now completed.

Theorem 2.5. Let $0<\beta<1$, and condition (1.3) be hold. If

$$
\sum_{n=N}^{\infty} q_{n}\left(1-M^{\alpha-1} p_{n+1-l} R_{n+1-k-l}^{\alpha-1}\right)^{\beta} R_{n+1-l}^{\beta}=\infty,
$$

and

$$
\lim _{n \rightarrow \infty} \sup \sum_{s=N}^{n-1}\left[K^{\beta-1} A_{s+1} q_{s}\left(1-\frac{K^{\alpha-1} p_{s+1-l} A_{s+1-k-l}^{\alpha}}{A_{s+1-l}^{\alpha}}\right)^{\beta}-\frac{1}{4 a_{s} A_{s+1}}\right]=\infty
$$

hold for all constants $M>0$, and $K>0$, then every solution of equation (1.1) is oscillatory.

Proof. Proceeding as in the proof of Theorem 2.4, we see that two cases of Lemma 2.1 hold for all $n \geq N$.

Case(1). From equation (1.1) and (2.1), we have (2.9). Further using (2.2) in (2.9), we obtain

$$
\Delta\left(a_{n} \Delta z_{n}\right)+q_{n}\left(1-M^{\alpha-1} p_{n+1-l} R_{n+1-l-k}^{\alpha-1}\right)^{\beta} R_{n+1-l}^{\beta}\left(a_{n+1-l} \Delta z_{n+1-l}\right)^{\beta} \leq 0
$$

for $n \geq N$. Let $w_{n}=a_{n} \Delta z_{n}>0$, and from the inequality (2.21) we see that $\left\{w_{n}\right\}$ is a positive solution of the inequality

$$
\Delta w_{n}+q_{n}\left(1-M^{\alpha-1} p_{n+1-l} R_{n+1-l-k}^{\alpha-1}\right)^{\beta} R_{n+1-l}^{\beta} w_{n+1-l}^{\beta} \leq 0 .
$$

Since $0<\beta<1$, we see that by condition (2.19) and a Theorem 1 of [5], the inequality (2.22) has no positive solution, a contradiction.

Case(2). Proceeding as in the proof of Case(2) of Theorem 2.4, we obtain (2.12). Define

$$
u_{n}=\frac{a_{n} \Delta z_{n}}{z_{n}}, n \geq N .
$$

Then $u_{n}<0$ for all $n \geq N$, and from (2.23), we obtain

$$
\begin{aligned}
\Delta u_{n} & =\frac{\Delta\left(a_{n} \Delta z_{n}\right)}{z_{n+1}}-\frac{a_{n} \Delta z_{n}}{z_{n} z_{n+1}} \Delta z_{n} \\
& \leq-q_{n}\left(1-\frac{K^{\alpha-1} p_{n+1-l} A_{n+1-k-l}^{\alpha}}{A_{n+1-l}^{\alpha}}\right)^{\beta} \frac{z_{n+1}^{\beta}}{z_{n+1}}-\frac{u_{n}^{2}}{a_{n}} .
\end{aligned}
$$


Since $z_{n}$ is positive and decreasing there is a constant $K>0$ such that $z_{n} \leq K$ for all $n \geq N$. Using the last inequality in (2.24), we have

$$
\Delta u_{n} \leq-\frac{q_{n}}{K^{1-\beta}}\left(1-\frac{K^{\alpha-1} p_{n+1-l} A_{n+1-l-k}^{\alpha}}{A_{n+1-l}^{\alpha}}\right)^{\beta}-\frac{u_{n}^{2}}{a_{n}}, n \geq N .
$$

Multiplying the last inequality by $A_{n+1}$ and then summing the resulting inequality from $N$ to $n-1$, we obtain

$$
\begin{aligned}
A_{n} u_{n}-A_{N} u_{N} & +\sum_{s=N}^{n-1} \frac{u_{s}}{a_{s}}+\sum_{s=N}^{n-1} \frac{u_{s}^{2}}{a_{s}} A_{s+1} \\
& +\sum_{s=N}^{n-1} \frac{A_{s+1} q_{s}}{K^{1-\beta}}\left(1-\frac{K^{\alpha-1} p_{s+1-l} A_{s+1-l-k}^{\alpha}}{A_{s+1-l}^{\alpha}}\right)^{\beta} \leq 0
\end{aligned}
$$

which yields

$$
\sum_{s=N}^{n-1}\left[K^{\beta-1} A_{s+1} q_{s}\left(1-\frac{K^{\alpha-1} p_{s+1-l} A_{s+1-k-l}^{\alpha}}{A_{s+1-l}^{\alpha}}\right)^{\beta}-\frac{1}{4 a_{s} A_{s+1}}\right] \leq 1+A_{N} u_{N}
$$

when using (2.14). This contradicts (2.20) as $n \rightarrow \infty$, and the proof is now completed.

\section{Examples}

In this section, we present two examples to illustrate the main results.

Example 3.1. Consider a second order neutral difference equation

$$
\Delta\left(n(n+1) \Delta\left(x_{n}+\frac{1}{3 n^{2}} x_{n-2}^{3}\right)\right)+n^{3} x_{n-1}^{3}=0, n \geq 1 .
$$

Here $a_{n}=n(n+1), p_{n}=\frac{1}{3 n^{2}}, q_{n}=n^{3}, k=2, l=2, \alpha=\beta=3$. A simple calculation shows that $A_{n}=\frac{1}{n}$, and $R_{n}=\frac{n-1}{n}$. By taking $\rho_{n} \equiv 1$, one can see that the conditions (2.7) and (2.8) are satisfied, and hence by Theorem 2.4, every solution of equation (3.1) is oscillatory.

Example 3.2. Consider a second order neutral difference equation

$$
\Delta\left(2^{n} \Delta\left(x_{n}+\frac{1}{4^{n}} x_{n-1}^{3}\right)\right)+4^{n} x_{n-1}^{1 / 3}=0, n \geq 1 .
$$

Here $a_{n}=2^{n}, p_{n}=\frac{1}{4^{n}}, q_{n}=4^{n}, k=1, l=2, \alpha=3$ and $\beta=1 / 3$. Since $A_{n}=\frac{1}{2^{n-1}}, R_{n}=\frac{2^{n}-2}{2^{n}}$, and it is easy to verify that all conditions of Theorem 2.5 are satisfied, and hence every solution of equation (3.2) is oscillatory.

Remark 3.3. The results obtained in this paper extend some of the results in $[6,7,9,10]$ for $\alpha>1$. Also the results of [9] cannot be applied to equations (3.1) and (3.2) since $\sum_{n=1}^{\infty} \frac{1}{a_{n}}<\infty$. Hence our results generalize and extend some of the results reported in the literature.

Acknowledgement: The authors thank the reviewer for his/her useful corrections and suggestions which improved the content of the paper.

\section{References}

[1] R.P.Agarwal, Difference Equations and Inequalities: Theory, Methods and Applications, Second Edition, Marcel Dekker, New York, 2000. 
[2] R.P.Agarwal, M.Bohner, S.R.Grace, D.O'Regan, Discrete Oscillation Theory, Hindawi Pub. Corp., New York, 2005.

[3] X.Liu, Oscillation of solutions of neutral difference equations with a nonlinear term, J.Comp.Math.Appl., 52(2006), $439-448$.

[4] M.Migda and J.Migda, Oscillatory and asymptotic properties of solutions of even order neutral difference equations, J.Differ.Eqn.Appl., 15(2009), 1077-1084.

[5] X.H.Tang and Y.J.Liu, Oscillation for nonlinear delay difference equations, Tamkang J.Math., 32(2001), 275-280.

[6] E.Thandapani, Z.Liu, R.Arul and P.S.Raja, Oscillation and asymptotic behavior of second order difference equations with nonlinear neutral term, Appl.Math.E-Notes, 4(2004), 59-67.

[7] E.Thandapani, S.Pandian and R.K.Balasubramanian, Oscillation of solutions of nonlinear neutral difference equations with nonlinear neutral term, Far East J.Appl.Math., 15(2004), 47-62.

[8] E.Thandapani and P.M.Kumar, Oscillation and nonoscillation of nonlinear neutral delay difference equation, Tamkang J.Math., 38(2007), 323-333.

[9] M.K.Yildiz and H. Öğünmez, Oscillation results of higher order nonlinear neutral delay difference equations with a nonlinear neutral term, Hacettepe J.Math.Stat., 43(2014), 809-814.

[10] Z.Zhang, J.Chen and C.Zhang, Oscillation of solutions for second order nonlinear difference equations with nonlinear neutral term, Comput.Math.Appl., 41(2001), 1487-1494. 